\title{
CARACTERÍSTICA DOS CATETERES E DE CRIANÇAS PORTADORAS DE DOENÇA ONCOHEMATOLÓGICA*
}

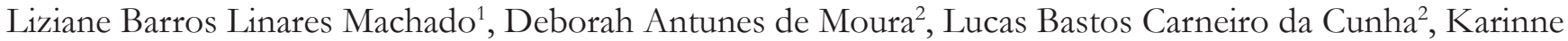 \\ Cristinne da Silva Cunha ${ }^{3}$
}

\begin{abstract}
RESUMO: O objetivo deste estudo foi identificar o perfil clínico de crianças/adolescentes com doença oncohematológica e as características dos cateteres utilizados por estes. Estudo descritivo, com abordagem quantitativa de 78 fichas de vigilância epidemiológica. O cenário foi um ambulatório de oncohematologia pediátrica, os dados foram coletados em agosto de 2015. Observou-se que 32 (59\%) dos portadores de cateter totalmente implantado eram do sexo masculino, e sete (53\%) dos portadores de cateter epicutâneo eram do sexo feminino, a idade variava entre 10 e 16 anos, o principal diagnóstico foi leucemia linfoide aguda em ambos. A média de utilização do cateter totalmente implantado foi 303 dias, enquanto do cateter epicutâneo foi 55 dias, o término do tratamento é o principal motivo de retirada de ambos. A falta de preenchimento de informações nas fichas foi limitador desta pesquisa. Concluímos que estes dispositivos são eficazes e seguros para tratamento quimioterápico.
\end{abstract}

DESCRITORES: Criança; Hematologia; Cateteres; Enfermagem; Quimioterapia.

\section{CHARACTERISTIC OF CATHETERS AND OF CHILDREN WITH ONCOHEMATOLOGICAL DISEASE}

\begin{abstract}
The objective in this study was to identify the clinical profile of children/adolescents with onco-hematological diseases and the characteristics of the catheters they use. Descriptive study with quantitative approach of 78 epidemiological surveillance forms. The scenario was a pediatric onco-hematology outpatient clinic. The data were collected in August 2015. It was observed that $32(59 \%)$ of the patients using a totally implanted catheter were male, and seven (53\%) patients with a peripherally inserted central venous catheter were female. Ages ranged between 10 and 16 years. The main diagnosis was acute lymphoid leukemia in both. The mean length of use of the totally implanted catheter was 303 days, against 55 days for the peripherally inserted central venous catheter. The end of the treatment is the main motive for the withdrawal of both. The lack of completed information in the forms limited this research. In conclusion, these devices are effective and safe for chemotherapeutic treatment.
\end{abstract}

DESCRIPTORS: Child; Hematology; Catheters; Nursing; Chemotherapy.

\section{CARACTERÍSTICA DE LOS CATÉTERESY DE NIÑOS PORTADORES DE ENFERMEDAD ONCO-HEMATOLÓGICA}

RESUMEN: La finalidade del estudio fue identificar el perfil clínico de niños/adolescentes con enfermedad onco-hematológica, así como las características de los catéteres utilizados por ellos. Estudio descriptivo, con abordaje cuantitativo de 78 fichas de registro epidemiológicas. El contexto fue el de un ambulatorio de onco-hematología pediátrica y los datos fueron obtenidos en agosto de 2015. Se observó que 32 (59\%) de los portadores de catéter totalmente implantado eran de sexo masculino, y que siete (53\%) de los portadores de catéter epicutáneo eran de sexo feminino; la edad variaba entre 10 y 16 años; el diagnóstico principal fue el de leucemia linfoide aguda en ambos sexos. La media de utilización del catéter totalmente implantado fue de 303 días, mientras del catéter epicutáneo fue de 55 días; el término del tratamiento es el principal motivo de retirada de ambos. La falta de informaciones en las fichas de registro fue un factor limitador de esta investigación. Se concluye que estes dispositivos son eficaces y seguros para tratamiento quimioterápico.

DESCRIPTORES: Niño; Hematología; Catéteres; Enfermería; Quimioterapia.

*Artigo extraído da dissertação intitulada: "Ocorrência de infecção em cateter totalmente implantado na oncohematologia pediátrica: levantamento de estratégias para controle". Universidade Federal do Estado do Rio de Janeiro, 2015.

${ }^{1}$ Enfermeira. Mestranda em Saúde e Tecnologia. Enfermeira Chefe do Serviço de Onco Hematologia Pediátrica do Hospital Federal da Lagoa. Rio de Janeiro, RJ, Brasil.

${ }^{2}$ Discente de Enfermagem. Universidade Federal do Estado do Rio de Janeiro. Rio de Janeiro, RJ, Brasil.

${ }^{3}$ Enfermeira. Doutora em Neuroimunologia. Docente de Enfermagem e de Pós-graduação em Saúde e Tecnologia no Espaço Hospitalar na Universidade Federal do Estado do Rio de Janeiro. Rio de Janeiro, RJ, Brasil.

Autor Correspondente:

Liziane Barros Linares Machado

Universidade Federal do Estado do Rio de Janeiro

Rua da Passagem, 130 - 22.290-031 - Rio de Janeiro, RJ, Brasil

E-mail: lizblinares@yahoo.com.br
Recebido: $12 / 09 / 2016$

Finalizado: 19/12/2016 


\section{- INTRODUÇÃO}

Os tumores infantis são geralmente de natureza embrionária, do sistema reticuloendotelial, do sistema nervoso central, do tecido conectivo e de vísceras ${ }^{(1)}$. São considerados como um conjunto de neoplasias que ocorre em menores de 15 anos, as faixas etárias pediátricas mais precoces ( 0 a 4 anos) são as mais propensas ao desenvolvimento de câncer, e, de um modo geral, as neoplasias comuns em adultos raramente ocorrem em crianças ${ }^{(1)}$.

O câncer infantil não pode ser visto como uma simples doença e deve ser encarado como uma gama de diferentes malignidades ${ }^{(2)}$.

Tanto no Brasil quanto em países desenvolvidos, o câncer já representa a primeira causa de morte por doença entre crianças e adolescentes de 1 a 19 anos (7\% do total), considerando todas as regiões ${ }^{(3)}$.

Ao longo dos anos de 2016 e 2017, estão previstos 12.600 novos casos de câncer em crianças e adolescentes no Brasil. As regiões mais afetadas são: Sudeste e Nordeste variando entre 6.050 e 2.750 novos casos, seguidas pela região Sul com 1.320, Centro-Oeste 1.270 e Norte com $1.210^{(3)}$.

Durante o tratamento ambulatorial da criança ou adolescente com doença oncohematológica, são necessárias inúmeras internações hospitalares e é de extrema importância, para o sucesso do seu tratamento, o estabelecimento de um acesso venoso vascular de longa permanência.

Portanto, um acesso venoso de longa permanência que promova confiança durante a administração de quimioterápicos é primordial no manejo do paciente com câncer. Os acessos utilizados por estes pacientes são conhecidos no meio hospitalar como: cateter venoso central totalmente implantado (CVC-TI), cateter venoso central semi-implantado (CVC-SI) e cateter central de inserção periférica (CCIP).

Na instituição onde este trabalho foi realizado, os cateteres mais comumente utilizados são o cateter totalmente implantado e o cateter central de inserção periférica, por esta razão nos deteremos a estes dois dispositivos.

Nesse setor, parte dos pacientes não estão internados no hospital. Eles chegam para atendimento ambulatorial, permanecem em suas residências e só vão para a realização do tratamento quimioterápico, consultas e manutenção de seus dispositivos.

O cateter totalmente implantado é também conhecido no meio hospitalar como Ports ou Porta-caths. São dispositivos de borracha siliconizada cuja extremidade distal se acopla a uma câmera puncionável que se aloja no tecido subcutâneo da região torácica sobre uma protuberância óssea ${ }^{(4)}$.

Esses cateteres destinam-se à infusão de quimioterápicos, soro, antibióticos, sangue e seus componentes, nutrição parenteral, contrastes, ou qualquer outro fluido endovenoso e coleta de amostra sanguínea para exames laboratoriais. A punção desses cateteres deve ser feita por profissional treinado, preferencialmente enfermeiro. Possuem boa aceitação entre os pacientes, pois não requerem cuidados domiciliares e sua interferência em autoimagem é mínima ${ }^{(4)}$.

O cateter venoso central de inserção periférica (CCIP) é um dispositivo intravenoso que é introduzido por meio de uma veia superficial que progride até a veia cava superior ou inferior, que permite a infusão de soluções com extremos de pH e osmolaridade, como drogas vesicantes ou irritantes muito utilizadas no decorrer do tratamento oncohematológico ${ }^{(5)}$.

A decisão do tipo de cateter a ser utilizado na criança ou adolescente não cabe apenas à equipe multiprofissional responsável pela assistência, mas também ao familiar responsável, que deverá estar de acordo com o tipo de cateter escolhido e o local de inserção. Ao permitir a participação do paciente e seu familiar na implantação do cateter, tem-se a possibilidade de reduzir os impactos de alteração da autoimagem corporal, e permitir que a criança ou adolescente tenha conhecimento das limitações impostas pelo tratamento ${ }^{(6)}$.

Este estudo é relevante pois traz dois diferentes tipos de dispositivos utilizados para a realização do tratamento quimioterápico em crianças e adolescentes, relata sobre um implantado cirurgicamente 
por médicos e outro implantado pelo enfermeiro e mostra que desde que indicados e manipulados de forma correta eles conseguem cumprir com o objetivo proposto que é proporcionar um tratamento ininterrupto e seguro para o paciente, mostra ainda que o CCIP pode ser um grande aliado no tratamento dos nossos pacientes e isso serve de embasamento para que a sua utilização cresça na prática da oncologia pediátrica como cateter de primeira escolha e não como um cateter secundário.

Neste contexto o objetivo desta pesquisa foi identificar o perfil clínico de crianças/adolescentes com doença oncohematológica e as características dos cateteres utilizados para realização do seu tratamento.

\section{MÉTODO}

Trata-se de um estudo descritivo, retrospectivo, realizado em agosto de 2015, com abordagem quantitativa de dados secundários coletados de 78 fichas de vigilância epidemiológica, de 60 crianças que tiveram durante seu tratamento um ou mais cateteres.

O cenário escolhido para a busca de dados foi um ambulatório de oncohematologia pediátrica, localizado em um hospital federal no município do Rio de Janeiro. A coleta de dados do estudo foi baseada nos itens que constam na ficha de vigilância epidemiológica. Esta ficha foi desenvolvida pela equipe da oncohematologia pediátrica em conjunto com a comissão de controle de infecção hospitalar $\mathrm{CCIH}$ do referido hospital, como estratégia para melhor controle da manipulação dos cateteres.

Foram incluídas no estudo as fichas de crianças e adolescentes que durante o seu acompanhamento ambulatorial utilizaram o cateter totalmente implantado e/ou cateter venoso central de inserção periférica. Foram utilizadas as fichas dos cateteres que não estavam mais em uso, ou seja, que já haviam sido retirados no recorte temporal de julho de 2011 a abril de 2015.

Os dados coletados foram referentes aos diferentes tipos de manipulação dos dispositivos, principais problemas apresentados pelos mesmos, além de dados referentes ao seu usuário como idade, sexo, patologia, dentre outros.

Foram excluídas fichas de crianças e adolescentes que tiveram mais de dois cateteres durante seu tratamento oncohematológico. Foram selecionadas e analisadas 78 de um total de 90 fichas de vigilância epidemiológica.

Os resultados obtidos na coleta de dados foram organizados em uma planilha, foi realizado uma análise descritiva das variáveis a partir da comparação com a literatura e os dados então foram computados em tabelas.

O presente estudo foi submetido ao Comitê de Ética em Pesquisa da UNIRIO e foi aprovado com o parecer número: 1.213.979. Como não houve contato direto do pesquisador com as crianças e adolescentes que utilizaram os cateteres, foi solicitada a dispensa do uso do termo de consentimento livre e esclarecido.

\section{O RESULTADOS}

De acordo com os dados, observou-se que eram utilizados dois tipos de cateteres durante o tratamento no ambulatório de oncohematologia: o cateter totalmente implantado com $81 \%$, $(n=63)$ e o cateter central de inserção periférica com 19\%, (n=15).

Constatamos que $59 \%(n=32)$ da amostra dos portadores de cateter totalmente implantado era do sexo masculino, com prevalência de faixa etária entre 11 a 16 anos com 18\% ( $\mathrm{n}=10)$. Quanto ao diagnóstico médico desses portadores do cateter do sexo masculino, o destaque vai para a Leucemia Linfoide Aguda (LLA) com 37\% $(n=20)$. Já os portadores do cateter totalmente implantado do sexo feminino configuraram $41 \%(n=22)$, destacando a faixa etária de maior representatividade de 0 a 10 anos $15 \%$ ( $n=0$ ito). O diagnóstico médico de maior incidência entre o sexo feminino também foi a LLA com 28\% ( $n=15)$ (Tabela 1$)$. 
Tabela 1 - Distribuição quantitativa e relativa do sexo, idade e diagnóstico dos portadores do cateter totalmente implantado e CCIP. Rio de Janeiro, RJ, Brasil, 2016

\begin{tabular}{|c|c|c|c|c|c|c|c|c|c|c|c|c|c|c|}
\hline \multicolumn{15}{|l|}{ CVC-TI } \\
\hline \multirow{2}{*}{\multicolumn{3}{|c|}{ Sexo/Idade }} & \multicolumn{3}{|c|}{0 a 5} & \multicolumn{2}{|c|}{6 a 10} & \multicolumn{2}{|c|}{11 a 16} & \multicolumn{3}{|c|}{ sem informação } & \multicolumn{2}{|c|}{ total } \\
\hline & & & 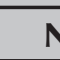 & & & $\mathbf{N}$ & $\%$ & $\mathbf{N}$ & $\%$ & $\mathbf{N}$ & & & $\mathbf{N}$ & $\%$ \\
\hline \multicolumn{3}{|l|}{ Feminino } & 8 & & & 8 & 15 & 0 & 0 & 6 & & & 22 & 41 \\
\hline \multicolumn{3}{|l|}{ Masculino } & 8 & & & 4 & 8 & 10 & 18 & 10 & & & 32 & 59 \\
\hline \multicolumn{3}{|l|}{ Total } & 1 & & & 12 & 22 & 10 & 18 & 16 & & & 54 & 100 \\
\hline \multicolumn{15}{|l|}{ CCIP } \\
\hline \multirow{2}{*}{\multicolumn{3}{|c|}{ Sexo/Idade }} & \multicolumn{3}{|c|}{0 a 5} & \multicolumn{2}{|c|}{6 a 10} & \multicolumn{2}{|c|}{11 a 16} & \multicolumn{3}{|c|}{ sem informação } & \multicolumn{2}{|c|}{ total } \\
\hline & & & $\Lambda$ & & & $\mathbf{N}$ & $\%$ & $\mathbf{N}$ & $\%$ & $\mathbf{N}$ & & & $\mathbf{N}$ & $\%$ \\
\hline \multicolumn{3}{|l|}{ Feminino } & 1 & & & 2 & 15 & 4 & 30 & 0 & & & 7 & 53 \\
\hline \multicolumn{3}{|l|}{ Masculino } & 1 & & & 0 & 0 & 5 & 38 & 0 & & & 6 & 47 \\
\hline \multicolumn{3}{|l|}{$\begin{array}{l}\text { Total } \\
\text { CVC-TI }\end{array}$} & 2 & & & 0 & 0 & 9 & 69 & 0 & & & 13 & 100 \\
\hline \multirow[t]{2}{*}{$\begin{array}{l}\text { Sexo/ } \\
\text { Diagnóstico }\end{array}$} & \multicolumn{2}{|c|}{ LMA } & \multicolumn{2}{|c|}{ LLA } & \multicolumn{2}{|c|}{$\begin{array}{l}\text { Linfoma } \\
\text { Hodgkin }\end{array}$} & \multicolumn{2}{|c|}{$\begin{array}{c}\text { Linfoma não } \\
\text { Hodgkin }\end{array}$} & \multicolumn{2}{|c|}{ Histiocitose } & \multicolumn{2}{|c|}{ Mielodisplasia } & \multicolumn{2}{|c|}{ Total } \\
\hline & $\mathbf{N}$ & $\%$ & $\mathbf{N}$ & $\%$ & $\mathbf{N}$ & $\%$ & $\mathbf{N}$ & $\%$ & $\mathbf{N}$ & $\%$ & $\mathbf{N}$ & $\%$ & $\mathbf{N}$ & $\%$ \\
\hline Feminino & 4 & 7 & 15 & 28 & 1 & 2 & 0 & 0 & 1 & 2 & 1 & 2 & 22 & 41 \\
\hline Masculino & 2 & 4 & 20 & 37 & 3 & 5 & 7 & 13 & 0 & 0 & 0 & 0 & 32 & 59 \\
\hline Total & 6 & 11 & 35 & 65 & 4 & 7 & 7 & 13 & 1 & 2 & 1 & 2 & 54 & 100 \\
\hline \multicolumn{15}{|l|}{ CCIP } \\
\hline \multirow[t]{2}{*}{$\begin{array}{l}\text { Sexo/ } \\
\text { Diagnóstico }\end{array}$} & & & & & & $\begin{array}{l}\text { foma } \\
\text { Igkin }\end{array}$ & $\begin{array}{r}\operatorname{Linf} \\
\mathrm{HC}\end{array}$ & $\begin{array}{l}\text { a não } \\
\text { kin }\end{array}$ & Hist & itose & Miel & plasia & & tal \\
\hline & $\mathbf{N}$ & $\%$ & $\mathbf{N}$ & $\%$ & $\mathbf{N}$ & $\%$ & $\mathbf{N}$ & $\%$ & $\mathbf{N}$ & $\%$ & $\mathbf{N}$ & $\%$ & $\mathbf{N}$ & $\%$ \\
\hline Feminino & 0 & 0 & 3 & 23 & 4 & 30 & 0 & 0 & 0 & 0 & 0 & 0 & 7 & 53 \\
\hline Masculino & 0 & 0 & 4 & 30 & 2 & 17 & 0 & 0 & 0 & 0 & 0 & 0 & 6 & 47 \\
\hline Total & 0 & 0 & 7 & 53 & 6 & 47 & 0 & 0 & 0 & 0 & 0 & 0 & 13 & 100 \\
\hline
\end{tabular}

Fonte: Dissertação de mestrado Machado LBL, 2016

No que diz respeito à idade dos pacientes portadores de CCIP, nota-se que a prevalência de faixa etária entre 11 a 16 anos, 68\% (n=nove) do conjunto, em relação ao diagnóstico observa-se que a LLA é preponderante, com $53 \%$ ( $n=$ sete) do total.

No que diz respeito ao local de inserção do cateter totalmente implantado (Tabela 2), o hemitórax direito foi o local mais utilizado com $50 \%(n=39)$, seguido pelo hemitórax esquerdo com $14 \%(n=11)$. O membro superior esquerdo e direito e a jugular direita também foram usados, porém, em menor frequência.

Já os dados levantados para inserção do CCIP revelam que, o membro superior direito foi o mais utilizado com $11 \%$ ( $n=$ nove), e em segundo foi o membro superior esquerdo com $6 \%$ ( $n=$ cinco).

Tabela 2 - Distribuição quantitativa e relativa dos locais de inserção do cateter totalmente implantado e do CCIP. Rio de Janeiro, RJ, Brasil, 2016 (continua)

\begin{tabular}{|c|c|c|c|c|c|c|c|c|c|c|c|c|c|c|}
\hline & \multicolumn{2}{|c|}{ HTD } & \multicolumn{2}{|c|}{ HTE } & \multicolumn{2}{|c|}{ MSD } & \multicolumn{2}{|c|}{ MSE } & \multicolumn{2}{|c|}{ Jugular D } & \multicolumn{2}{|c|}{$\begin{array}{c}\text { Sem } \\
\text { informação }\end{array}$} & \multicolumn{2}{|c|}{ Total } \\
\hline & $\mathbf{n}$ & $\%$ & $\mathbf{n}$ & $\%$ & $\mathbf{n}$ & $\%$ & $\mathbf{n}$ & $\%$ & $\mathbf{n}$ & $\%$ & $\mathbf{N}$ & $\%$ & $\mathbf{n}$ & $\%$ \\
\hline CVC-TI & 39 & 50 & 11 & 14 & 4 & 5 & 6 & 8 & 0 & 0 & 3 & 4 & 63 & 81 \\
\hline CCIP & 0 & 0 & 0 & 0 & 9 & 11 & 5 & 6 & 1 & 1 & 0 & 0 & 15 & 19 \\
\hline Total & 39 & 50 & 11 & 14 & 13 & 17 & 11 & 14 & 1 & 1 & 3 & 4 & 78 & 100 \\
\hline
\end{tabular}




\begin{tabular}{lccc} 
& Média (dias) & Menor tempo (dias) & Maior tempo (dias) \\
\hline CVC-TI & 303 & 11 & 928 \\
\hline CCIP & 55 & 9 & 154
\end{tabular}

Fonte: Dissertação de mestrado Machado LBL, 2016

Analisando a presença de possíveis intercorrências relacionadas ao uso do cateter totalmente implantado (Tabela 3), notou-se que $84 \%(n=53)$ da amostra estudada não apresentou nenhuma intercorrência com o cateter. Este fato pode estar relacionado ao início da implantação da ficha de vigilância no setor onde a equipe estava sendo capacitada para seu preenchimento, este fato é uma lacuna em nosso estudo.

Porém, uma intercorrência presente foi a hipertermia representando 3\% ( $n=$ dois), os outros 13\% $(\mathrm{n}=\mathrm{um})$ foram relacionadas igualmente às seguintes intercorrências: flebite, secreção pulmonar oriunda do mau posicionamento do cateter em região pleural, cateter acotovelado em subclávia, bacteremia, infecção, extravazamento, derrame pleural, exteriorização do fronth, exteriorização da agulha.

Foram analisados os motivos que levaram à retirada do cateter totalmente implantado, e o de maior porcentagem foi o não identificado com $36 \%(n=23)$. Isto pode estar relacionado às primeiras fichas que foram utilizadas para a função de monitorar este cateter, pois havia ainda uma adaptação da equipe com o novo instrumento, o término do tratamento aparece com $27 \%(n=17)$, seguido por óbito com 14\% ( $n=$ nove), a infecção aparece em quarto lugar representando um valor de $9 \%$ ( $n=$ seis), dos cateteres retirados, a infecção era diagnosticada após o resultado da coleta de hemocultura do cateter positivar.

Tabela 3 - Distribuição quantitativa e relativa das intercorrências e dos motivos de retirada do cateter totalmente implantado. Rio de Janeiro, RJ, Brasil, 2016

\begin{tabular}{|c|c|c|c|c|c|}
\hline Motivo de retirada & & & Intercorrências & & \\
\hline & $\mathbf{n}$ & $\%$ & & $\mathbf{n}$ & $\%$ \\
\hline Óbito & 9 & 14 & Flebite & 1 & 2 \\
\hline Infecção & 6 & 9 & Secreção pulmonar & 1 & 2 \\
\hline Mau funcionamento & 1 & 2 & Cateter acotovelado em subclávia & 1 & 2 \\
\hline Término do tratamento & 17 & 27 & Bacteremia & 1 & 2 \\
\hline Edema & 1 & 2 & Extravazamento & 1 & 2 \\
\hline Bacteremia & 1 & 2 & Derrame Pleural & 1 & 2 \\
\hline Término da quimioterapia & 3 & 5 & Exteorização do fronth & 1 & 2 \\
\hline Granuloma com exposição do porth & 1 & 2 & Hipertermia & 2 & 3 \\
\hline Cateter em ventrículo & 1 & 2 & Exteriorização da agulha & 1 & 2 \\
\hline Transplante & 1 & 2 & Sem intercorrências & 53 & 84 \\
\hline Não especificado & 23 & 36 & Infecção & 1 & 2 \\
\hline Total & 64 & 100 & Total & 64 & 100 \\
\hline
\end{tabular}

Fonte: Dissertação de mestrado Machado LBL, 2016

Em relação aos motivos que levaram ao manuseio do cateter totalmente implantado (Tabela 4), a coleta de sangue foi o motivo que mais se destaca, com um total de 1.573 manipulações, seguido pela troca de equipo que resultou em 1.373 manipulações do cateter.

O número de ativação e desativação do cateter alcançou um total de 1.119 manipulações.

Outros motivos também foram identificados, porém em menor frequência, como instalação de quimioterapias e manutenção do mesmo que é realizada uma vez a cada 30 dias para troca da solução de heparina, que é utilizada para manter a permeabilidade do cateter. 
Tabela 4 - Distribuição quantitativa e relativa dos motivos de manuseio no cateter totalmente implantado. Rio de Janeiro, RJ, Brasil, 2016

\begin{tabular}{lccccc} 
& Total & Média & Moda & Mediana & Desvio Padrão \\
\hline Quimioterapia & 364 & 6 & 1 & 6 & 5,543 \\
\hline Coleta de Sangue & 1573 & 19 & 13 & 19 & 19,712 \\
\hline Manutenção & 348 & 6 & 1 & 6 & 5,820 \\
\hline Lock Therapy & 28 & 2 & 2 & 2 & 0,743 \\
\hline Outros & 281 & 5 & 2 & 5 & 5,634 \\
\hline Número de Ativações/ Desativações & 119 & 15 & 12 & 15 & 12,389 \\
\hline Troca de Equipos & 1373 & 14 & 10 & 14 & 21,064 \\
\hline Quantidade Total de Uso & 1119 & 15 & 12 & 15 & 12,389
\end{tabular}

Fonte: Dissertação de mestrado Machado LBL, 2016

A Tabela 5 apresenta dados referentes ao CCIP, e com relação ao calibre, observa-se que o de maior escolha é o de $n^{\circ} 03$, com $80 \%$ do total $(n=12)$. Quanto à finalidade de manipulação, percebe-se que o CCIP foi mais utilizado para coleta de sangue $(n=127)$, seguido de quimioterapia $(n=51)$.

Para realizarmos a ativação do cateter, é necessária a retirada da solução de heparina do mesmo através da aspiração, após é administrado um flush de soro fisiológico, habilitando assim o mesmo para uso.

A partir de uma única ativação pode se fazer coleta de sangue, quimioterapia, manutenção, entre outras manipulações, por isso o número de ativações/desativações do cateter aparece inferior do que a união dos demais.

Os motivos de retirada mais significativos são o término da quimioterapia e a obstrução, ambos com $20 \%(n=3)$ cada. Há também 13\% ( $n=$ dois) de cateteres que aparecem sem descrição sobre o motivo da retirada. Houve somente um caso de infecção, sendo $7 \%(n=1)$.

Tabela 5 - Distribuição quantitativa em relação ao calibre do cateter, finalidades da manipulação e motivos de retirada do CCIP. Rio de Janeiro, RJ, Brasil, 2016 (continua)

\begin{tabular}{|c|c|c|c|c|c|}
\hline \multirow[t]{2}{*}{ Calibre } & & \multicolumn{4}{|c|}{ Total } \\
\hline & & \multicolumn{2}{|c|}{$\mathbf{N}$} & \multicolumn{2}{|c|}{$\%$} \\
\hline 3 & & \multicolumn{2}{|c|}{12} & \multicolumn{2}{|c|}{80} \\
\hline 4 & & \multicolumn{2}{|c|}{2} & \multicolumn{2}{|r|}{13} \\
\hline Sem informação & & \multicolumn{2}{|c|}{1} & \multicolumn{2}{|r|}{7} \\
\hline Total & & \multicolumn{2}{|c|}{15} & \multicolumn{2}{|c|}{100} \\
\hline Motivos de Manuseio & Total & Média & Moda & Mediana & Desvio Padrão \\
\hline Quimioterapia & 51 & 3,4 & 1 & 3 & 2,58 \\
\hline Coleta de Sangue & 127 & 8,46 & 4 & 5 & 7,56 \\
\hline Manutenção & 48 & 3,2 & 0 & 2 & 3,54 \\
\hline Ativações/ Desativações & 105 & 7 & 7 & 6 & 5,55 \\
\hline \multirow[t]{2}{*}{ Motivo de retirada } & & & & \multicolumn{2}{|c|}{ Total } \\
\hline & & & & $\mathbf{N}$ & $\%$ \\
\hline Infecção & & & & 1 & 7 \\
\hline Tracionamento do Cateter & & & & 1 & 7 \\
\hline Furo no óstio de inserção & & & & 1 & 7 \\
\hline Extensão cortada & & & & 1 & 7 \\
\hline Hiperemia no trajeto & & & & 1 & 7 \\
\hline Solicitação médica & & & & 1 & 7 \\
\hline
\end{tabular}




\begin{tabular}{lcc}
\hline Presença de trombo & 1 & 7 \\
\hline Término da quimioterapia & 3 & 20 \\
\hline Obstrução & 3 & 20 \\
\hline Sem Informação & 2 & 13 \\
\hline Total & 15 & 100
\end{tabular}

Fonte: Dissertação de mestrado Machado LBL, 2016

\section{- DISCUSSÃO}

O perfil dos portadores do cateter totalmente implantado pesquisado demonstra semelhanças com a literatura já existente. De acordo com a Tabela 1,59\% $(n=32)$ dos usuários do cateter totalmente implantado eram do sexo masculino, com faixa etária dos 11 aos 16 anos e tendo o diagnóstico médico de maior prevalência a Leucemia Linfoide Aguda (LLA) com 37\% $(n=20)$. Uma amostra realizada em 61 prontuários de crianças e adolescentes que haviam passado por um procedimento cirúrgico para implantação de um cateter totalmente implantado, revelou que os adolescentes entre 10 a 17 anos eram os que mais utilizavam o cateter, desta amostra $59 \%$ também eram do sexo masculino ${ }^{(7)}$.

Outro estudo com 1.472 pacientes com diagnóstico de leucemia linfoide aguda, revelou que 883 $(56,59 \%)$ destes pacientes eram do sexo masculino e $639(43,41 \%)$ do sexo feminino ${ }^{(8)}$. Este resultado pode estar relacionado ao fato da leucemia linfoide aguda ser mais prevalente no sexo masculino do que no feminino.

Com relação ao CCIP, a maioria dos pacientes que o utilizaram é do sexo feminino e o diagnóstico preponderante também é a LLA. Esse resultado está de acordo com um estudo em oncohematologia pediátrica em relação ao diagnóstico, porém difere em relação ao sexo ${ }^{(9)}$ além deste, um trabalho distinto mostra a preponderância do sexo masculino na utilização do cateter ${ }^{(10)}$.

Outro estudo díspar mostra que o diagnóstico em que o CCIP foi mais utilizado foi o de Linfoma Não-Hodgkin ${ }^{(11)}$.

As leucemias são o tipo de câncer infantil mais comum em menores de 15 anos na maioria das populações, correspondendo entre $25 \%$ e $35 \%$ de todos os tipos. Ainda nesta faixa etária os linfomas, tumores do sistema nervoso central e do sistema simpático, rabdomiossarcomas, tumor de Wilms, retinoblastomas e tumores ósseos são frequentemente diagnosticados ${ }^{(1-2)}$.

Há uma maior predominância dos casos de LLA em comparação as outras doenças hematológicas ${ }^{(8)}$. Observamos que os estudos realizados pelos autores acima estão de acordo com os dados levantados por nossa pesquisa em que a LLA e o sexo masculino aparecem em maior porcentagem em relação às outras patologias oncohematológicas.

No que se refere à análise demonstrada na Tabela 2, observou-se que o hemitórax direito (HTD) foi o local mais utilizado para a implantação do cateter totalmente implantado com $50 \%$ ( $n=39$ ), seguido pelo hemitórax esquerdo (HTE) com 14\% ( $n=11)$. Já em um estudo realizado em 61 cateteres revelou que normalmente estes são inseridos na região infraclavicular, neste estudo observamos que a veia de primeira opção foi a subclávia direita com $68,9 \%$ da amostra, seguido da subclávia esquerda com 3,3\% e veia axilar direita representando $1,6 \%$ deste total ${ }^{(7)}$.

Já o CCIP no que tange ao local de inserção, o local mais selecionado foi o membro superior direito, seguido do membro superior esquerdo e da veia jugular direita, o que é um resultado semelhante a outro estudo ${ }^{(12)}$. Além deste, há um estudo que mostra a preponderância da seleção ao membro superior direito ${ }^{(13)}$.

Uma pesquisa mostrou que o membro superior direito tem maior taxa de sucesso de inserção na primeira tentativa do que o membro superior esquerdo ${ }^{(14)}$. Contudo, alguns estudos mostram a seleção do braço ${ }^{(15-16)}$ não dominante e sendo esse a maioria o membro superior esquerdo ${ }^{(14)}$. 
A literatura traz que a veia jugular direita é uma das últimas opções para inserção devido à dificuldade de fixação, maior risco de haver migração do cateter e de infecção. Já a veia jugular externa direita é preferencial à esquerda, pois há risco de ascender para veia jugular interna ao invés da veia cava. Essa dissertação corrobora com o resultado de somente uma punção ter sido feita na jugular direita, encontrado na presente pesquisa ${ }^{(15-17)}$.

Outra pesquisa mostra que os pacientes afirmam estarem mais satisfeitos com o cateter quando ele está localizado abaixo do ombro ${ }^{(18)}$ como nos membros superiores. Esse dado está de acordo com os 93\% dos cateteres CCIP terem sido inseridos nos membros superiores na presente pesquisa.

A Tabela 2 nos revela a análise de intercorrências relacionadas ao uso do cateter totalmente implantado, $84 \%(n=53)$ da amostra estudada não apresentou nenhuma intercorrência com o cateter. No entanto, uma intercorrência presente foi a hipertermia representando $3 \%(n=2)$. Os outros $13 \%$ $(n=1)$ relacionavam-se igualmente às seguintes intercorrências: flebite, secreção pulmonar oriunda do mal posicionamento do cateter em região pleural, cateter acotovelado em subclávia, bacteremia, infecção, extravazamento, derrame pleural, exteriorização do fronth, exteriorização da agulha.

A literatura mostra que as complicações relacionadas aos cateteres podem ser classificadas em: precoces, que ocorrem entre as primeiras 48 horas a sete dias pós implantação do cateter ou até mesmo no momento de sua primeira utilização, e as tardias, que ocorrem após este período ${ }^{(7)}$. Entretanto, esse mesmo estudo não apresenta dados suficientes que permitam a classificação dessas complicações.

Uma pesquisa realizada com cateteres profundos revelou que complicações como a obstrução aparecem em maior destaque, seguido por infecção, extravasamento, trombose e deslocamento do cateter $^{(19)}$.

Uma outra pesquisa revela que dos 71 cateteres implantados, a infecção foi detectada 23 vezes, levando à remoção de oito dispositivos ${ }^{(20)}$. A conduta adotada para o manejo dessas complicações infecciosas previa a confirmação da infecção através da comparação entre a hemocultura colhida do cateter e a hemocultura colhida por via periférica. Após a identificação do sítio de infecção e da identificação do microorganismo é que se era estabelecido a antibioticoterapia adequada.

Análise realizada por um estudo evidenciou que as principais complicações relacionadas ao cateter foram complicações tardias incluindo bacteremia generalizada com $(8,43 \%)$, seguido por inflamação local da pele e do tecido subcutâneo $(1,2 \%)$, trombose venosa $(2,41 \%)$, a migração do cateter $(1,2 \%)$ e necrose da pele sobre a câmara do porth $(2,41 \%)^{(21)}$.

Um dispositivo intravenoso como o CVC-TI pode causar danos ao paciente, principalmente quando este está internado, dentre estes danos a infecção que pode gerar sepse e a descontinuidade do tratamento ${ }^{(22)}$.

Também na Tabela 2, foi possível identificar os motivos que resultaram na retirada do cateter. O motivo de maior destaque não foi identificado e configurou $36 \%(n=23)$, seguido pelo término do tratamento com $27 \%(n=17)$, e pelo óbito com $14 \%(n=9)$. Já a infecção aparece com $9 \%(n=6)$. As complicações como obstrução aparecem com relevância entre os estudos citados, já em nossa pesquisa esta complicação não aparece, porém, a infecção aparece como um importante motivo de retirada deste dispositivo.

Com a análise dos dados obtidos, foi possível detectar os motivos que resultaram no manuseio do cateter totalmente implantado, demonstrado na Tabela 4. A coleta de sangue foi o motivo de manuseio do cateter de maior expressão, seguido pela troca de equipo. Já a ativação e desativação do cateter e a instalação de quimioterapias aparecerem em menores proporções. Na literatura, a antissepsia da pele, a punção do cateter totalmente implantado e o curativo foram os motivos de manuseio do cateter totalmente implantado mais citados em pesquisa ${ }^{(20)}$.

Porém outros cuidados de enfermagem com o cateter aparecem no que diz respeito à manipulação, como assistência pré e pós-operatória, administração de medicamentos, curativos e manutenção da permeabilidade ${ }^{(20)}$.

Quanto à finalidade de uso do CCIP, observou-se que a maioria de sua utilização foi para coletas de sangue. Porém, outro estudo mostra que o cateter foi mais utilizado para administração de 
quimioterápicos ${ }^{(9)}$, que foi a segunda maior utilização do presente estudo.

Nos resultados obtidos, a maior parte dos motivos de retirada foi o término da quimioterapia e obstrução, o que coincide com outra pesquisa que mostra o término do tratamento como maior motivo de retirada ${ }^{(10)}$.

Entretanto, outros estudos ${ }^{(9-11)}$ revelam que o maior motivo de retirada foi a infecção, o que difere do nosso, pois a infecção foi encontrada em um único caso. O resultado encontrado é bastante positivo já que se trata de pacientes imunossuprimidos ${ }^{(12)}$.

Vários são os motivos de manipulação destes dois diferentes dispositivos, porém quando estes são contabilizados é que temos uma dimensão do quanto eles são manipulados para os diferentes procedimentos, tanto pela equipe médica quanto pelos enfermeiros(as) e do quanto uma complicação precoce ou tardia pode ser evitada com os devidos cuidados prestados.

\section{CONCLUSÃO}

Ao analisar os dados oriundos das fichas de vigilância epidemiológica, conseguimos levantar o perfil da nossa clientela e as principais características dos cateteres utilizados por elas para a realização de seu tratamento.

Foi identificado que 32 (59\%) dos portadores de cateter totalmente implantado são do sexo masculino, com maior incidência na faixa etária entre 11-16 anos e a patologia que mais acomete estes pacientes é a LLA.

Já os portadores do CCIP, do sexo feminino correspondem a 53\% ( $\mathrm{n}=$ sete), no que diz respeito à idade dos pacientes, nota-se que estão na faixa etária de 11 a 16 anos, em relação ao diagnóstico observa-se que a LLA é preponderante.

Os resultados deste estudo sugerem que o CCIP é seguro para administração de quimioterapia por vários ciclos, pois a média de 55 dias de utilização do cateter já o caracteriza como um cateter de longa duração, permitindo a possibilidade de usá-lo até o término do tratamento. Além disso, a baixa taxa de infecção mostra como a utilização do CCIP é confiável e eficaz no tratamento oncohematológico pediátrico.

Como limitador da pesquisa, aparece a falta de preenchimentos de algumas informações nas fichas, ocasionando um prejuízo na precisão dos dados, fazendo assim se inferir resultados que podiam ter sido mais precisos.

Apesar das vantagens que estes dispositivos oferecem, eles devem ser manipulados por profissionais treinados para a realização da técnica de maneira correta, pois ocasionalmente a falta de destreza pode estar associada a complicações, como infecção, obstrução, flebite, extravasamento quimioterápico, entre outras.

Concluímos que as baixas taxas de complicação obtidas neste estudo confirmam a segurança e conveniência do uso dos CVC-TI e do CCIP em crianças e adolescentes portadores de doenças oncohematológicas que fazem uso prolongado de quimioterapia.

\section{REFERÊNCIAS}

1. Braga PE, Latorre MRDO, Curado MP. Câncer na infância: análise comparativa da incidência, mortalidade e sobrevida em Goiânia (Brasil) e outros países. Cad. Saúde Pública. [Internet] 2002;18(1) [acesso em 10 jun 2016$].$ Disponível: http://dx.doi.org/10.1590/S0102-311X2002000100004.

2. Instituto Nacional do Câncer (INCA). Tipos de câncer. Leucemia. [Internet] 2016 [acesso em 15 ago 2016$].$ Disponível: http://www2.inca.gov.br/wps/wcm/connect/inca/portal/home.

3. Instituto Nacional do Câncer (INCA). Tipos de câncer. Infantil. [Internet] 2016 [acesso em 01 set 2016$].$ Disponível: http://www2.inca.gov.br/wps/wcm/connect/tiposdecancer/site/home/infantil. 
4. Bonassa EMA, Gato MIR. Terapêutica Oncológica para enfermeiros e farmacêuticos. $4^{\mathrm{a}}$ ed. São Paulo: Atheneu; 2012.

5. Camara D. Minimizing risks associated with peripherally inserted central catheters in the NICI. MCN Am J Matern Child Nurs. [Internet] 2001;26(1) [acesso em 05 ago 2016]. Disponível: http://journals.Iww.com/mcnjournal/ Abstract/2001/01000/Minimizing_Risks_Associated_With_Peripherally.5.aspx.

6. Martins FTM, de Carvalho EC. A percepção do paciente referente a ser portador de um cateter de longa permanência. Rev. esc. enferm. USP. [Internet] 2008;42(3) [acesso em 09 ago 2016]. Disponível: http://dx.doi. org/10.1590/S0080-62342008000300016.

7. Ortoloni L, Gasparino RC, Traldi MC. Complicações associadas ao uso de cateter totalmente implantável em crianças e adolescentes. Rev. Bras. Cancerol. [Internet] 2013;59(1) [acesso em 12 jul 2016]. Disponível: http:// pesquisa.bvsalud.org/portal/resource/pt/lil-722815.

8. Pereira WV. Aspectos epidemiológicos, biotipologia e evolução do tratamento da leucemia linfocítica aguda na infância e adolescência no Rio Grande do Sul [tese]. São Paulo (SP): Universidade de São Paulo; 2010.

9. Bergami CMC, Monjardim MAC, Macedo CR. Utilização do cateter venoso central de inserção periférica (PICC) em oncologia pediátrica. Reme, Rev. Min. Enferm. [Internet] 2012;16(4) [acesso em 08 jun 2016]. Disponível: http:// www.dx.doi.org/S1415-27622012000400009.

10. Hasanbegović E, Sabanović S, Majstorović S. Peripheral insertion of central catheters in the treatment of pediatric patients with leukemia. Med Arh. [Internet] 2000;54(5-6) [acesso em 08 jun 2016]. Disponível: http:// www.ncbi.nlm.nih.gov/pubmed/11219913.

11. Frachiolla NS, Todisco E, Bilancia A, Gandolfi S, Mancini V, Marbello L, et al. Peripherally Inserted Central Catheters (PICCs) Implantation in the Clinical Management of Oncohematologic Patients: Results of a Large Multicenter, Retrospective Study of the REL Group (Rete Ematologica Lombarda - Lombardy Hematologic Network, Italy). American Society of Hematology. [Internet] 2015;126(23) [acesso em 09 mai 2016]. Disponível: http://www.bloodjournal.org/content/126/23/5611.

12. Roberto P, Madeira F, Oliveira E, Carreira C, Carreira M, Soares F. Central venous line placement is not compromised by the choice between different insertion sites. Study performed in cardiovascular surgery patients. Rev Port Cir Cardiotorac Vasc. [Internet] 2013;20(1) [acesso em 08 jun 2016]. Disponível: http://www. ncbi.nlm.nih.gov/pubmed/24511578.

13. Evans RS, Sharp JH, Linford LH, Lloyd JF, Tripp JS, Jones JP, et al. Risk of symptomatic DVT associated with peripherally inserted central catheters. Chest. [Internet] 2010;138(4) [acesso em 08 jun 2016]. Disponível: http:// dx.doi.org/10.1378/chest.10-0154.

14. Jeon EY, Cho YK, Yoon DY, Hwang JH. Which arm and vein are more appropriate for single-step, nonfluoroscopic, peripherally inserted central catheter insertion?. J Vasc Access [Internet] 2016;17(3) [acesso em 08 jun 2016]. Disponível: http://dx.doi.org/10.5301/jva.5000506.

15. McGill RL, Tsukahara T, Bhardwaj R, Kapetanos AT, Marcus RJ. Inpatient venous access practices: PICC culture and the kidney patient. J Vasc Access. [Internet] 2015;16(3) [acesso em 08 jun 2016]. Disponível: http://dx.doi. org/10.5301/jva.5000340.

16. Delarbre B, Dabadie A, Stremler-Lebel N, Jolibert M, Cassagneau P, Lebel S, et al. Introduction of the use of a pediatric PICC line in a French University Hospital: review of the first 91 procedures. Diagn Interv Imaging. [Internet] 2014;95(3) [acesso em 08 jun 2016]. Disponível: http://dx.doi.org/10.1016/j.diii.2013.05.004.

17. Sociedade Brasileira de Enfermeiros de Terapia Intensiva (SOBETI). Curso de Qualificação em Inserção, Utilização e Cuidados com o cateter venoso Cental de Inserção Periférica - CCIP- Neonatologia/Pediatria. São Paulo; 2004.

18. Polak JF, Anderson D, Hagspiel K, Mungovan J. Peripherally inserted central venous cathters: factors affecting patient satisfaction. AJR Am J Roentgenol. [Internet] 1998;170(6) [acesso em 08 jun 2016]. Disponível: http://dx.doi. org/10.2214/ajr.170.6.9609182.

19. de Souza GS, Rocha PRS, dos Reis PED, Vasques CI. Manuseio de cateter venoso central de longa permanência em pacientes portadores de câncer. R. Enferm. Cent. O. Min. [Internet] 2013;3(1) [acesso em 10 jun 2016]. 
Disponível: http://dx.doi.org/10.19175/recom.v0i0.340.

20. Vasques $\mathrm{Cl}$, dos Reis PED, de Carvalho EC. Manejo do cateter venoso central totalmente implantado em pacientes oncológicos: revisão integrative. Acta paul. enferm. [Internet] 2009;22(5) [acesso em 02 jun 2016]. Disponível: http://dx.doi.org/10.1590/S0103-21002009000500016.

21. Patel GS, Jain K, Kumar R, Strickland AH, Pellegrini L, Slavotinek J, et al. Comparison of peripherally inserted central venous catheters (PICC) versus subcutaneously implanted port-chamber catheters by complication and cost for patients receiving chemotherapy for non-haematological malignancies. Support care cancer. [Internet] 2014;22(1) [acesso em 02 set 2016]. Disponível: http://dx.doi.org/ 10.1007/s00520-013-1941-1.

22. de Oliveira TF, Rodrigues MCS. Enfermagem na prevenção de infecção em cateter totalmente implantado no paciente oncológico. Cogitare Enferm. [Internet] 2016;21(2) [acesso em 02 set 2016]. Disponível: http://dx.doi. org/10.5380/ce.v21i2.45523. 